\title{
Augmented Reality und Virtual Reality
}

\author{
Matthias Knoll (D) - Stefan Stieglitz (i)
}

Angenommen: 27. Januar 2022 / Online publiziert: 15. Februar 2022

(C) Der/die Autor(en) 2022

Beim Stichwort Virtual Reality (VR) und Augmented Reality (AR) könnte der erste Gedanke Filmen, Spielen oder neuesten Social-Media-Aktivitäten, wie etwa der vor kurzem begonnenen Entwicklung von Metaverse gelten. Filme wie „Avatar“ (VR) oder das Spiel „Pokemon“ (AR) sind heute allgemein bekannt. Doch die Ursprünge liegen lange vor dieser Zeit. Zwar ist es immer schwierig, den Ursprung eines Begriffs oder einer Technologie exakt zu verorten, schließlich sind viele Erfindungen evolutionär entstanden. Doch lässt sich der Begriff Virtual Reality bereits auf die Jahre 1967/68 zurückführen. Damals entwickelte Ivan Sutherland, ein Spezialist für Computergrafik, das erste „Headset“. Aufgrund seines Gewichts und seiner Abmessungen konnte es nicht getragen werden, vielmehr glich es eher den feststehenden Messapparaturen aus der Augenheilkunde und soll daher mitunter scherzhaft auch „Damoklesschwert“ genannt worden sein. Unabhängig von Fragen der Abmessungen und des Gewichts mussten für seine Entwicklung drei wesentliche Probleme gelöst werden: Zunächst bedarf es eines interaktiven Computer-Grafikprogramms. Bislang dominierte die Stapelverarbeitung (Batch-Betrieb) die IT. Im zweiten Schritt musste eine nicht prozedurale Programmiersprache für die Darstellung der Graphik entwickelt werden. Im dritten Schritt musste, da Objekte visualisiert werden sollten, ein objektorientiertes Softwaresystem geschaffen werden. Sutherland gelang es, Antworten auf diese Fragen zu finden. Die fertige Lösung wurde anschließend von der NASA erfolgreich für Trainingszwecke genutzt. Astronauten konnten so Arbeiten im All vorab üben. Doch dann mussten weitere fast 15 Jahre vergehen. Der uns

\footnotetext{
Matthias Knoll $(\bowtie)$

Hochschule Darmstadt, Darmstadt, Deutschland

E-Mail: matthias.knoll@h-da.de

Stefan Stieglitz

Digitale Kommunikation und Transformation, Universität Duisburg-Essen, Duisburg, Deutschland

E-Mail: stefan.stieglitz@uni-due.de
} 
heute geläufige Begriff Virtual Reality wurde erstmals in dem 1982 erschienenen Roman „The Judas Mandala“ des Autors Damien Francis Broderick erwähnt. Ebenfalls in diesem Jahr erschien der von Disney produzierte Film „Tron“. Und 1984 erlaubte der Roman „Cybermancer“ einen erweiterten Blick auf das Konzept der virtuellen Realität - und was daraus entstehen könnte ${ }^{1}$.

Es ist ein überaus interessantes, herausforderndes, vieldimensionales und inhaltlich spannendes Themenfeld. Dass die NASA die von Sutherland entwickelte Lösung 1968 produktiv einsetzte, zeigt, dass das Thema nicht nur für Gaming und Film relevant ist, im Gegenteil. In den letzten Jahren hat die Bedeutung von AR- und VRTechnologien und darauf basierenden Lösungen stark zugenommen. AR und VR werden branchenübergreifend und in vielfältiger Form in Unternehmen, Organisationen und in der Bildung eingesetzt. Immer neue Anwendungskontexte kommen hinzu. Doch wie genau sehen die Optionen aus, wenn man über den Einsatz von AR oder VR für das eigene Unternehmen, in der Forschung oder im Lehrbetrieb nachdenkt?

Diese Ausgabe will mit ihren Beiträgen bei der Beantwortung dieser und weiterer Fragen unterstützen. Die Beiträge sollen dabei helfen, die für die jeweilige Organisation ,richtige“ Antwort zu finden und so eine klare eigene Positionierung im Themenkreis Augmented und Virtual Reality zu ermöglichen. Hierzu ordnet der Einführungsbeitrag zunächst in kompakter Form die Begriffe, gibt einen Überblick über die verschiedenen Anwendungskontexte, Chancen und Risiken und schließt mit Tipps für den Einstieg.

Anschließend widmen sich die weiteren Beiträge unterschiedlichen Aspekten. Um die erfreuliche Vielzahl der Einreichungen besser strukturieren zu können, haben wir Themenbereiche definiert und in nachfolgender Übersicht die Titel zur inhaltlichen Orientierung im weiteren Heft zusammengefasst. Informationen zu den Autorenteams finden Sie wie stets im Inhaltsverzeichnis und bei den Beiträgen selbst.

\section{Branchenneutrale Aspekte}

Die 15 Beiträge in dieser Rubrik befassen sich mit unterschiedlichen Aspekten des AR-/VR-Einsatzes, denen ein gewisser Grundlagencharakter oder eine gewisse Universalität gemeinsam ist. Entsprechend werden die Fragen dort zwar in der Regel anhand konkreter Problemstellungen diskutiert, lassen sich jedoch leicht auch auf andere Branchen übertragen.

\subsection{Visualisierung komplexer Sachverhalte, Modellierung und Simulation}

- Toy or Tool? Utilitaristischer und hedonischer Nutzen mobiler Augmented Reality-Apps

- Entwicklung einer VR-Umgebung zur Exploration von Process-Mining

\footnotetext{
${ }^{1}$ Reimann (2018) Wie Ivan Sutherland im Jahr 1968 die virtuelle Realität erfunden hat. https://filmpuls. info/ivan-sutherland-virtuelle-realitaet-vr sowie Hertel (2017) Die Geschichte der virtuellen Realität. https://www.vrnerds.de/die-geschichte-der-virtuellen-realitaet.
} 
- Augmented Reality-Systeme zur Unterstützung der Workflow-Ausführung - Entwicklung und praktische Anwendung einer Taxonomie

- Affordance-Experimentation: Eine Fallstudie zur Entwicklung von Virtual Reality Anwendungsfällen im Unternehmenskontext

- Kontextabhängige Rechtsvisualisierung mit Augmented Reality

\subsection{Wissensaustausch, Lehren und Lernen}

- Entwicklung und Einsatz von AR-Anwendungen in der Hochschule am Beispiel des Projekts „Augmented Learning“ an der Universität Duisburg-Essen

- AR/VR.nrw - Augmented und Virtual Reality in der Hochschullehre

- Der Einsatz von Virtual-Reality Lernszenarien für Softskills-Trainings

- Wissensmanagement in virtuellen Welten: Wissensgenerierung in Virtual RealityUmgebungen

\subsection{Kollaboration/Zusammenarbeit}

- Kollaboration mit Extended Reality Systemen, eine Kategorisierung

- Ausgestaltungs- und Anwendungspotenziale von Virtual und Augmented Reality Technologien im Kontext von Coworking Spaces

- Herausforderungen und Handlungsempfehlungen für die VR-basierte Zusammenarbeit mit digitalen Nomad*innen und anderen Remote-Arbeitenden

- AR- und Holografie-gestütztes Netzwerken als Alternative zum traditionellen Netzwerken vor Ort - ein multiperspektivischer Einblick

\subsection{Externe Kommunikation}

- Co-Creation in Virtual Reality: Immersion als Treiber des Kundenerlebnisses

- Zwischen Preisjägern, Datenschützern und Tech-Enthusiasten: Segmentierung des Virtual-Reality-Marktes am Beispiel Oculus

\section{Branchenbezogene Aspekte}

In diesen acht Beiträgen steht der konkrete Anwendungsfall in einer speziellen Branche im Vordergrund. Dennoch liefert auch diese Rubrik durchaus Impulse für vergleichbare Anwendungskontexte in benachbarten Einsatzgebieten.

\subsection{Industrie/Handel}

- Try before you buy - Akzeptanz von Virtual-Reality-Anwendungen zur Leistungsbeurteilung von Erfahrungsgütern 
- Virtual Reality als Trainingsmethode: Eine Laborstudie aus dem Industriebereich

- Use Cases für industrielle Anwendungen der Augmented Reality Technologie

- Die Rolle von 3D-Modellen im Wertschöpfungsprozess von physischen und Virtuellen Konsumgütern

- Anwendungsszenarien für AR in der Produktion: Use Cases und Technologielösungen

\subsection{Gesundheitswesen}

- ALiS: Entwicklung einer Designtheorie für Augmented Living Spaces zur erweiterten Autonomie älterer und kognitiv eingeschränkter Menschen

\subsection{Spiele- und Unterhaltungsindustrie}

- Technologieadoptionsstrategien von Medienunternehmen. Schnelligkeit, Zeitpunkt und Planungshorizonte von Investitionen und Akquisitionen in Augmented und Virtual Reality-Technologien

\subsection{Spezialanwendungen}

- Anforderungen sicherheitskritischer Dienste an Augmented Reality-Lösungen Wassertiefenmessung in einem Seehafen

Drei Rezensionen runden die Ausgabe ab. Die erste Rezension widmet sich einem Grundlagenbuch. Ralf Dörner et al. beleuchten in diesem Werk umfassend Grundlagen und Methoden der AR und VR und ergänzen damit diese Schwerpunktausgabe in idealer Weise. Die zweite Rezension wirft einen Blick auf den Einsatz von AR im E-Commerce, ein sicherlich nicht nur für den Handel interessantes Thema, denn auch die Investitionsgüterindustrie kann über B2B-Kanäle Potenziale erschließen.

Die dritte Rezension mag auf den ersten Blick für ein wissenschaftliches Journal ein wenig aus dem Rahmen fallen, darf aber mit Blick auf den künstlerischen Aspekt von AR und VR nicht fehlen. Uwe Post, ein bekannter Schriftsteller und Sachbuchautor, beschreibt in seinem Buch ein dystopisches Szenario. Wer über die notwendigen finanziellen Mittel verfügt, kann seinen Geist nach dem Tod in die Cloud laden und dort weiterleben und mit den Angehörigen Kontakt halten. Dass hier AR-/VR-Ansätze in geeigneter Form angewandt werden, erscheint konsequent und kann das Buch zum Gegenstand interessanter Diskurse machen.

Unser herzlicher Dank gilt allen Autorinnen und Autoren, die unter den aktuell weiterhin schwierigen Bedingungen ihr Wissen mit uns und Ihnen teilen und auf 
diesem Weg wertvolle Impulse für die Diskussion und Implementierung von ARund VR-Lösungen geben.

Wir wünschen Ihnen eine spannende Lektüre, aus der Sie viel Wissen mitnehmen können, und freuen uns über Ihr Lob, aber auch über Ihre Fragen, Anregungen und Kritik.

\section{Matthias Knoll und Stefan Stieglitz}

Funding Open Access funding enabled and organized by Projekt DEAL.

Open Access Dieser Artikel wird unter der Creative Commons Namensnennung 4.0 International Lizenz veröffentlicht, welche die Nutzung, Vervielfältigung, Bearbeitung, Verbreitung und Wiedergabe in jeglichem Medium und Format erlaubt, sofern Sie den/die ursprünglichen Autor(en) und die Quelle ordnungsgemäß nennen, einen Link zur Creative Commons Lizenz beifügen und angeben, ob Änderungen vorgenommen wurden.

Die in diesem Artikel enthaltenen Bilder und sonstiges Drittmaterial unterliegen ebenfalls der genannten Creative Commons Lizenz, sofern sich aus der Abbildungslegende nichts anderes ergibt. Sofern das betreffende Material nicht unter der genannten Creative Commons Lizenz steht und die betreffende Handlung nicht nach gesetzlichen Vorschriften erlaubt ist, ist für die oben aufgeführten Weiterverwendungen des Materials die Einwilligung des jeweiligen Rechteinhabers einzuholen.

Weitere Details zur Lizenz entnehmen Sie bitte der Lizenzinformation auf http://creativecommons.org/ licenses/by/4.0/deed.de. 